\title{
Urban Arterial Road Optimization and Design Combined with HOV Carpooling under Connected Vehicle Environment
}

\author{
Lina Mao $\mathbb{D}^{1},{ }^{1}$ Wenquan Li $\mathbb{D},{ }^{1}$ Pengsen Hu, ${ }^{1}$ Guiliang Zhou $\mathbb{D}^{2,3}$ Huiting Zhang, ${ }^{2}$ \\ and Xuanyu Zhou ${ }^{2}$ \\ ${ }^{1}$ School of Transportation, Southeast University, Nanjing 210096, China \\ ${ }^{2}$ Jiangsu Key Laboratory of Traffic and Transportation Security, Huaiyin Institute of Technology, Huaian 223003, China \\ ${ }^{3}$ School of Automotive and Traffic Engineering, Jiangsu University, Zhenjiang 212013, China
}

Correspondence should be addressed to Wenquan Li; seu_liwenquan@163.com and Guiliang Zhou; zglpaper@qq.com

Received 3 February 2019; Accepted 16 August 2019; Published 19 December 2019

Academic Editor: Jose E. Naranjo

Copyright (C) 2019 Lina Mao et al. This is an open access article distributed under the Creative Commons Attribution License, which permits unrestricted use, distribution, and reproduction in any medium, provided the original work is properly cited.

The HOV carpooling lane offers a feasible approach to alleviate traffic congestion. The connected vehicle environment is able to provide accurate traffic data, which could optimize the design of HOV carpooling schemes. In this paper, significant tidal traffic flow phenomenon with severe traffic congestion was identified on North Beijing road (bidirectional four-lane) and South Huaihai road (bidirectional six-lane) in Huai'an, Jiangsu Province. The historical traffic data of the road segments were collected through the connected vehicle environment facilities. The purpose of this study is to investigate the effect of adopting two HOV schemes (regular HOV scheme and reversible HOV carpooling scheme) on the urban arterial road under connected vehicle environment. VISSIM was used to simulate the proposed two HOV carpooling schemes at the mentioned road segment. The simulation results showed that the reversible HOV carpooling scheme could not only mitigate the traffic congestion caused by traffic tidal phenomenon but also improve the average speed and traffic volume of the urban arterial road segment, while the regular HOV scheme may exert a negative impact on the average speed and traffic volume on the urban arterial road segment.

\section{Introduction}

1.1. Development of the HOV Carpooling Scheme. Carpooling has gradually become an effective way to improve traveling efficiency and alleviate traffic congestion. In order to encourage carpooling behavior, China has issued some policies to promote the online carpooling services. Highoccupancy vehicle (HOV) lanes have been widely adopted in lots of cities in the world in order to reduce gas emission and mitigate traffic congestion [1]. To further improve the road capacity, it was recommended to introduce highoccupancy vehicle (HOV) carpooling lane into heavy traffic urban arterial road [2]. At present, the HOV lane has been widely adopted in the United States and Canada, which are two of the earliest countries introducing the HOV lane [3]. According to nearly 40 years of HOV lane practice, it could be found that the number of passengers delivered by HOV lane per unit time is significantly larger than that of ordinary lanes [4].
According to the literature, the HOV carpooling schemes could be divided into four categories as follows:

(1) Regular HOV carpooling scheme. Regular HOV carpooling scheme is commonly set on the inner lane of the road, and the direction of traffic is in accordance with ordinary lanes. It is isolated from ordinary lanes through road markings or a buffer area. The regular HOV lane is currently the most widely adopted scheme in the world.

(2) Reversible HOV carpooling scheme. In some of the cities, traffic flow on urban arterial shows a tidal phenomenon in the morning peak and evening peak. Reversible HOV lane refers to the HOV lane that can change the direction of the traffic during peak time. The direction of reversible HOV lane is in accordance with main traffic flow during the peak time, while at the nonpeak time, it runs the same direction as the ordinary lanes [5]. 
(3) Bidirectional isolated regular HOV carpooling scheme. The bidirectional isolated HOV lane is widely used in Europe and America, generally set in traffic generation areas like large residential areas and work areas. The traffic flow of such areas is generally $1300-1800$ vehicles/h. The inner lane of both directions of the traffic is set as the HOV lane. It is separated from ordinary lanes by a green belt or isolation barrier [6].

(4) Isolated reversible HOV carpooling scheme. The inner lane of minor traffic is set as HOV carpooling lane and is isolated from ordinary lanes through the physical facilities. The configuration of lanes in major traffic remained unchanged. The direction of the HOV lane in the minor traffic changes to the major traffic direction.

Several problems on setting HOV carpooling lane have been identified by researchers all over the world. Konishi and Mun [1] identify that the introducing HOV scheme may lead to poor effectiveness in easing traffic congestion caused by the low utilization of HOV lanes. Moreover, Dahlgren [7] states that introducing an extra regular lane rather than a HOV lane may have a better effect on mitigating traffic congestion under most of the circumstances. Pooler et al. [8] indicate that in some city that already has BRT lane, the HOV scheme may let those bus passengers choose to use less efficient carpools, which would worsen the air pollution and increase fuel consumption.

1.2. HOV Carpooling Scheme in China. Comparing to the developed countries, China is still in its early stage of adopting HOV lane. In 1997, Professor Bi Renzhong analyzed and summarized the development of HOV lane in different countries. He further analyzed the traffic situation in China and put forward the feasibility of implementing the $\mathrm{HOV}$ scheme in China. In 2003, Chen Wei and Chen Bailei proposed that the "HOV first" concept should be written into traffic laws and regulations [9]. In 2007, Zhan et al. adapted multiplechoice logit model to predict the effectiveness of introducing HOV lane [10]. In 2008, for the HOV lane, utilization rate is not high; Li Pengfei proposed that HOV lane could be incorporated into the bus transit lane. In 2010, Wang Weili first proposed the concept of rideshare [11]. With this, the concept of high-occupancy carpooling lane has emerged.

The first HOV lane in China was built at Xingyuan road in Wuxi, allowing the car with more than 3 passengers to access. Department of transportation in Shenzhen also put forward to introduce HOV carpooling lane on "four vertical and six horizontal" urban arterial road network, which mainly includes South China Sea Avenue, Nanping Urban Expressway, Xiangmi Lake road, and other urban arterial roads. The HOV carpooling scheme was introduced by expanding the arterial roads on both sides on the basis of the original six lanes. Those HOV carpooling lanes are opened all day. In Wuxi, Xingyuan road makes the left lane among six lanes as HOV carpooling lane shared with bus transit lane. These schemes are more suitable for urban arterial roads with sufficient reserved space in large cities but may not suitable for the road without reserved space to expand in some old cities.
In China, the HOV carpooling lane has improved traffic condition to some extent, but there are also some problems in practical application. The HOV carpooling lane on Shenzhen Binhai Avenue is set in the inner lane of the road which is connected to lots of interchanges and entrances, resulting in a large number of traffic conflicts. Xingyuan road in Wuxi is quite narrow. So that the bus transit lane and HOV lane were set as the same lane. When the bus leaves from the left lane to the right lane to stop at the bus stop, it will disturb the traffic on its right lanes, causing traffic congestion. In this case, the $\mathrm{HOV}$ carpooling lane exerts a negative impact on the traffic.

As the traffic situation in China is complicated, most of the urban arterial roads do not have sufficient spare space to expand. Thus, the isolated HOV carpooling scheme is not suitable for urban arterial roads in old cities [12]. The adoption of different types of HOV carpooling schemes requires an indepth analysis of historical traffic data, which could be obtained through the connected vehicle environment facilities.

\subsection{Improved HOV Carpooling Scheme under the Connected}

Vehicle Environment. From the literature, the main obstacles that weaken the effect of adopting the HOV carpooling scheme could be concluded as follows: (1) HOV lane conflicts with the bus lane; (2) the adopted type of HOV carpooling scheme does not match the original road configuration; and (3) the design of HOV scheme lacks accurate traffic data, which means the design scheme may not mitigate the traffic problem efficiently.

The improvements for HOV carpooling lane can be considered from the following aspects: (1) The bus should drive on the right lane of the road, and the car with more than 9 seats cannot access the HOV carpooling lane; (2) in order to avoid traffic congestion in one direction, the opening time of HOV carpooling lane should be set according to the regularity of traffic flow. Moreover, the traffic direction on HOV carpooling lane should be reversible according to the traffic volume in two directions; and (3) the connected vehicle environment facilities should be installed on the road in order to provide accurate traffic data.

In this paper, the North Beijing road (bidirectional fourlane) and South Huaihai road (bidirectional six-lane) in Huaian, Jiangsu Province, were selected as the object road section. Both of the urban arterial road sections have significant traffic tidal phenomenon, which leads to severe traffic congestion on the heavy traffic direction during the peak hour. The accurate historical traffic data are available through connected vehicle environment facilities. The design of the HOV carpooling schemes will be based on the travel pattern, traffic volume, average speed, and vehicle types in peak hour at the road segments. Considering the original road segments do not have enough space for expansion, two types of HOV carpooling schemes without isolation facilities were proposed: regular $\mathrm{HOV}$ carpooling scheme and reversible HOV carpooling scheme. VISSIM was used to simulate and evaluate the traffic condition after introducing the proposed schemes. The historical traffic data obtained from connected vehicle environment facilities were used to optimize the design of proposed schemes. 
This paper is organized as follows. In Section 2, we presented several evaluation and simulation methods of road segments. In Section 3, we proposed two types of HOV carpooling schemes based on the historical traffic data of the road segments obtained from connected vehicle environment facilities. In Section 4, VISSIM was used to investigate the effectiveness of introducing two types of proposed HOV carpooling schemes in the urban arterial road. The conclusion was drawn in Section 5.

\section{Evaluation and Simulation of Urban Arterial Roads Combined with HOV Carpooling under Connected Vehicle Environment}

\subsection{Investigation and Acquisition of Basic Data}

(1) Acquisition of traffic data. In this paper, the traffic data (traffic volume, average speed, etc.) are collected through either field survey or sensors under the connected vehicle environment built by the Key Laboratory of Transportation and Safety of Jiangsu Province.

(2) OD survey of the road segment. The OD survey is also called original destination survey; the investigation is based on traveler starting point and endpoint investigation of the individual traveler in a certain area. This method is used to analyze individual travel volume.

(3) Survey of vehicle type. Without any interference to normal traffic operations, the surveyors were deployed at a road intersection, rapidly identifying vehicle type and the number of passengers. The collected data could be used to calculate the proportion of different vehicle types.

(4) Survey of residents' rideshare willingness. The HOV carpooling lane could only be accessible by high occupation vehicles for a certain period of time. Carpooling vehicles might have more possibilities to access the HOV car-pooling lane. In order to verify whether the original roads are suitable to introduce HOV carpooling lanes, it is necessary to investigate residents' rideshare willingness. The survey was conducted by questionnaire, which accurately analyzes the residents' rideshare willingness with setting up HOV carpooling lanes.

\subsection{Evaluation of the Urban Arterial Roads with HOV Carpooling Lanes}

2.2.1. The Number of Passengers Passing through the Road Cross Section. The number of people passing the road cross section refers to the number of passengers carried by means of a vehicle at a certain road cross section in a fixed time [13]. The calculation is shown in Equation (1).

$$
P=\sum_{i=1}^{k} m_{i} * c_{i}
$$

where $m_{i}$ is the flow rate of vehicles $i$ and $c_{i}$ is the average number of passengers in the vehicle $i$.
2.2.2. The Average Travel Time of Each Person on the HOV Carpooling Lane. The average travel time of each person on the HOV carpooling lane is defined as the ratio of a total number of passengers to the total vehicle travel time in a road segment. The calculation is shown in Equation (2).

$$
T=\frac{\sum_{i=1}^{k} m_{i} * t_{i}}{\sum_{i=1}^{k} m_{i} * c_{i}}
$$

where $t_{i}$ is the travel time of the vehicle $i$.

2.2.3. The Average Travel Delay of Passenger. The average travel delay of the passenger is calculated as the ratio of total vehicle delay to the total number of passengers passing through the road cross section. The calculation is shown in Equation (3).

$$
D=\frac{\sum_{i=1}^{k} m_{i} * d_{i}}{\sum_{i=1}^{k} m_{i} * c_{i}}
$$

where $d_{i}$ is the travel delay of the vehicle $i$.

2.3. VISSIM Simulation of the Urban Arterial Roads with HOV Carpooling Lanes. After setting the parameters (road configuration, traffic volume, etc.), VISSIM can generate simulation results such as travel time, travel delay, and average travel time, so as to evaluate the effectiveness of introducing HOV carpooling scheme [14].

2.3.1. The Lane Selection Algorithm in VISSIM Simulation. In VISSIM, the lane selection module is integrated with lane management, and it is mainly determined by the number of passengers in the vehicle, simulation time, and current road traffic conditions [15]. Its decision-making process is based on the utility function. The calculation is shown in Equation (4).

$$
P_{(\text {Managed })}=1-\frac{e^{a * U_{\text {Managed }}}}{e^{a * U_{\text {Managed }}}+e^{a * U_{\text {UnManaged }}}}=1-\frac{1}{1+e^{a * \Delta U}},
$$

where $P$ represents the probability of using the managed lane, and $\Delta U$ is utility value. It consists of the cost of the travel time difference and the cost of lane management. The calculation is shown in Equation (5).

$$
\begin{aligned}
\Delta \text { Utility }= & \text { Cost coefficient } * \Delta \text { Cost } \\
& + \text { Time coefficient } * \Delta \text { Time. }
\end{aligned}
$$

2.3.2. VISSIM Simulation Model Construction. The aim of the simulation model is to establish a computational model which can reflect the actual traffic condition. It can optimize the design of the urban arterial road with HOV carpooling scheme and further evaluate its effectiveness [16]. When establishing the simulation model, we first identify the road segment; the basic data relevant to the area are investigated and acquired. After that, the parameters in the model are calibrated and verified. Finally, the simulation is carried out. According to the designed scheme, the HOV carpooling lane was introduced on the basis of the original road. The simulation results of original traffic data 
and simulation data of different test schemes were obtained. The average relative error evaluation was used to examine the validation. The calculation is shown in Equation (6).

$$
\bar{\lambda}=\frac{1}{k} \sum_{i=1}^{k} \frac{\left|q_{a i}-q_{b i}\right|}{q_{a i}}
$$

where $\bar{\lambda}$ is the average relative error; $k$ is the simulation times; $q_{a i}$ for the $i$-th simulation traffic flow; $q_{b i}$ for the $i$-th simulation of the actual traffic flow.

\section{A Case Study of the Urban Arterial Road Combined with HOV Carpooling under Connected Vehicle Environment-Take Huai'an City in Jiangsu Province as an Example}

Huaian is a city of obvious features that the urban has been expanding along the arterial roads, which is suitable for a case study. Basing on the traffic data, we designed two HOV carpooling schemes and established the simulation model through VISSIM to evaluate its effectiveness.

3.1. Location of the Investigated Road Segment. Specifically, we selected a road segment through the satellite map and field survey. A circle area with a diameter of one kilometer centered on Yong'an Bridge was selected as the object road segment, which is next to North Beijing road and South Huaihai road. The geographical relationship of Yong'an Bridge, North Beijing road, and South Huaihai road is shown in Figure 1.

\subsection{Traffic Data Acquisition}

3.2.1. Travel Pattern. As shown in Table 1, the survey found that for the Yongan Bridge segment at morning peak, traffic generation occurs mostly in residential area, and traffic attraction mainly occurs at schools and companies; however, at the evening peak, traffic generation occurs mostly at schools and companies, while the leisure entertainment and residential areas attract most of the traffic. The travel flow of the road segment between South Huaihai road and Meicheng road shows a tidal phenomenon. The village people rush to the South Station to work in the city in the morning peak, while they get off work and go back home at evening peak.

3.2.2. Traffic Volume. By extracting data from the connected vehicle system and field survey, the traffic volume on May 9, 2016, was collected. After processing the data, the hourly traffic volume in the morning and evening peak (namely 7:009:00 and 17:00-19:00) within the road segment is shown in Figures 2 and 3.

3.2.3. Average Speed. Similar to the traffic volume, the average speed is obtained through connected vehicle system and field survey. The processed average speed data are shown in Tables 2 and 3.

3.2.4. Vehicle Types in Peak Hour. In order to find out the number of vehicles using the HOV carpooling lane, we classify

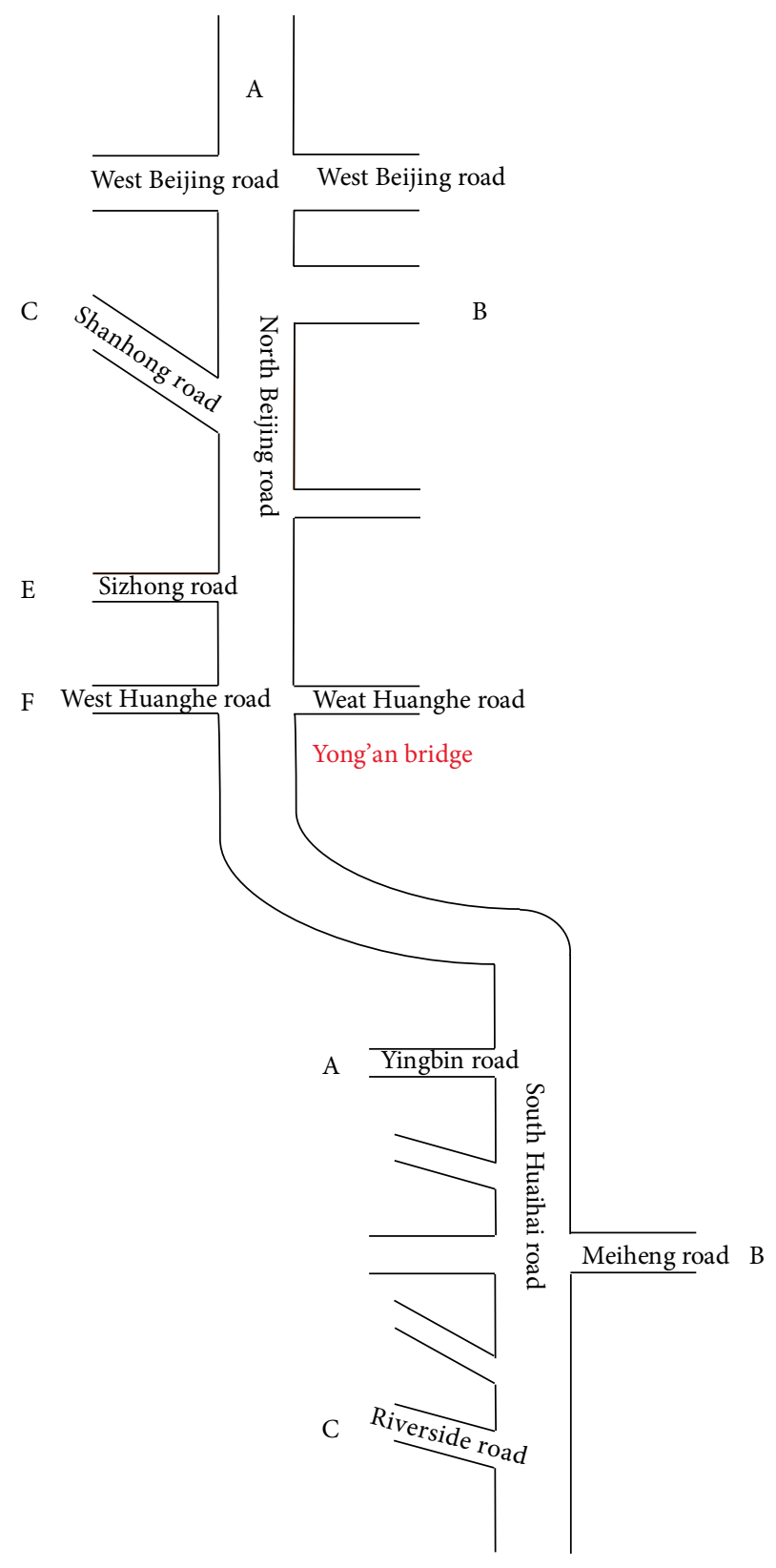

FIgURE 1: Geographical location of Yong'an Bridge, North Beijing road, and South Huaihai road.

the collected morning and evening peak hour traffic flow into four categories: sedan cars, medium buses, buses, and trucks. We further divided the sedan cars into three types according to the number of passengers on board.

From Table 4, it can be observed that in the surveyed road segment, the sedan car accounts for $93.36 \%$ of the total number of vehicles, while the bus, medium bus, and truck account for $6.64 \%$ of total number of vehicles.

3.3. HOV Carpooling Scheme Design. By analyzing the collected data, it can be found that during the morning peak (7:00-9:00), the southbound traffic flow is significantly greater than the northbound on North Beijing road, while on the South Huaihai road, the southbound traffic flow is 
TABLE 1: Travel pattern of North Beijing road.

\begin{tabular}{lcc}
\hline Site name & \multicolumn{1}{c}{$\begin{array}{c}\text { Travel time rule } \\
\text { Post meridiem (p.m.) }\end{array}$} \\
\hline $\begin{array}{l}\text { Rongyuan residence, Haojing residence, Jingyang garden, Yanhe garden, } \\
\text { Jianghuai residence, Hengtong building, Huaiyin district living area, Beijing } \\
\text { residence (second phase) }\end{array}$ & $6: 30-8: 30$ traffic generation & $6: 00-7: 30$ traffic attraction \\
$\begin{array}{l}\text { Wangying junior middle school, Kaiming middle school in Huaiyin district, } \\
\text { Wangying 1st middle school }\end{array}$ & $7: 00-8: 00$ traffic attraction & $6: 00-7: 00$ traffic generation \\
$\begin{array}{l}\text { Bank of construction dormitory, Taoyuan residence, Huaiwu apartment, } \\
\text { Fuqiang 9th residence, Zhongtian garden, Fuqiang village } \\
\begin{array}{l}\text { Xiba park, Fuhao garden, Hehua park, Huaiyin district stadium } \\
\text { Tianlai village, Haile KTV, Shaolaile KTV }\end{array}\end{array}$ & $6: 00-7: 30$ traffic generation & $6: 30-8: 30$ traffic attraction \\
\hline
\end{tabular}

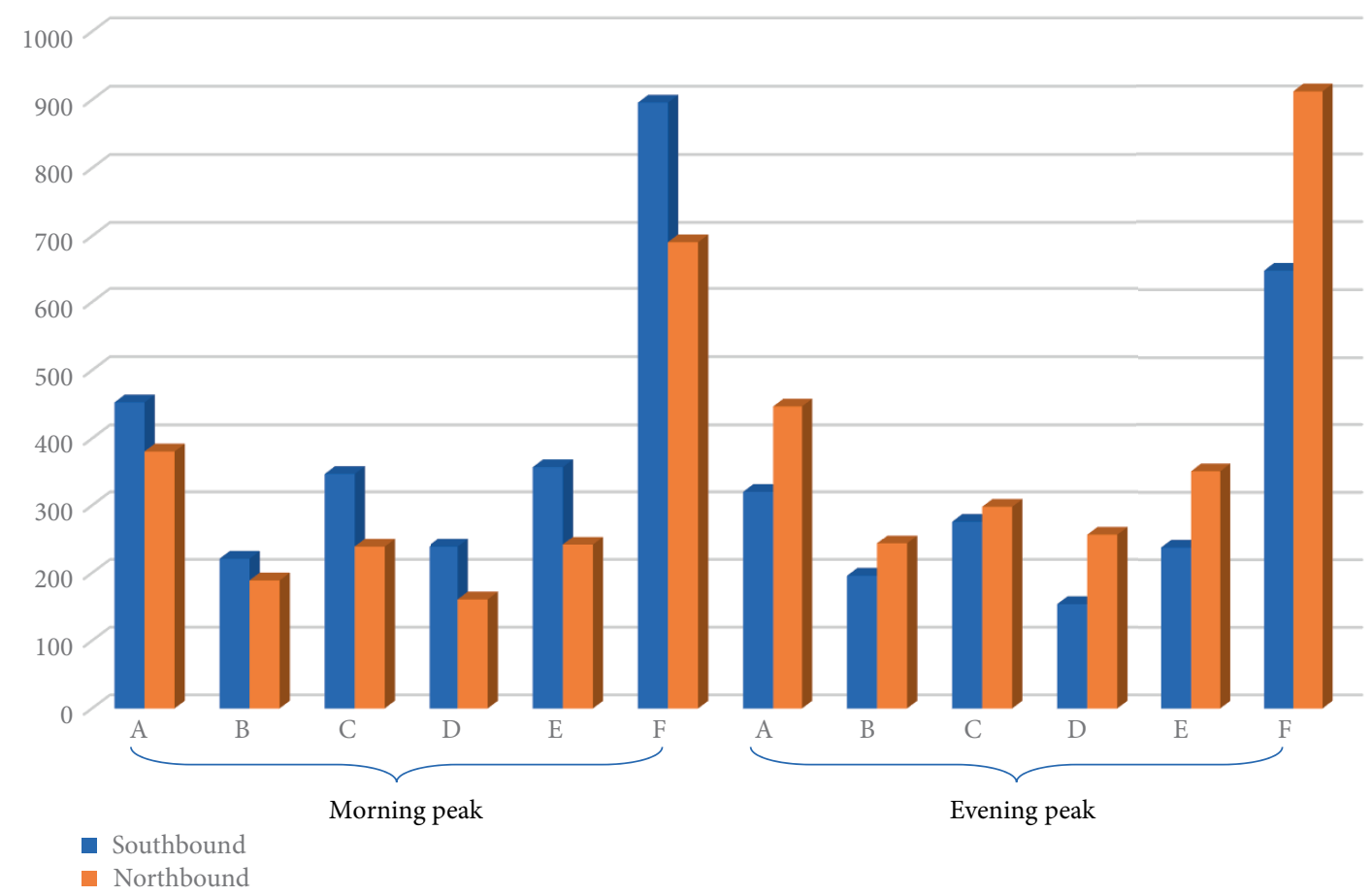

FIGURE 2: Hourly traffic volume of Yong'an Bridge segment in peak time.

less than the northbound. During the evening peak (17:0019:00), the southbound traffic flow is significantly less than the northbound on North Beijing road, while on the South Huaihai road, the situation is the opposite.

This paper proposed two HOV carpooling schemes in investigated road segment: regular HOV carpooling scheme and reversible carpooling scheme. Lane $\mathrm{B}$ and lane $\mathrm{C}$ of the North Beijing road were set as the reversible HOV carpooling lane, which are shown in Figures 4 and 5. The traffic of HOV lane B is changed from northbound to southbound in the morning peak, while the traffic of HOV carpooling lane $\mathrm{C}$ is changed from southbound to northbound in the evening peak. On South Huaihai road, lane C and lane D were set as reversible lane as well, which are shown in Figures 6 and 7. The traffic direction of HOV carpooling lane $\mathrm{D}$ is changed from southbound to northbound in the morning peak, while the traffic direction of lane $\mathrm{C}$ is changed from northbound to southbound in the evening peak.

(1) HOV Carpooling Schemes on North Beijing road.

\section{VISSIM Simulation and Evaluation of the Improved Road Segment}

\subsection{VISSIM Simulation}

4.1.1. Simulation of Road Configuration. VISSIM was adopted to establish a simulated intersection between South Huaihai road and East Meicheng road. The bus route, bus station, and proportion of different vehicle types were set in VISSIM according to the collected data. The simulation diagram of the selected road segment is presented in Figure 8. 


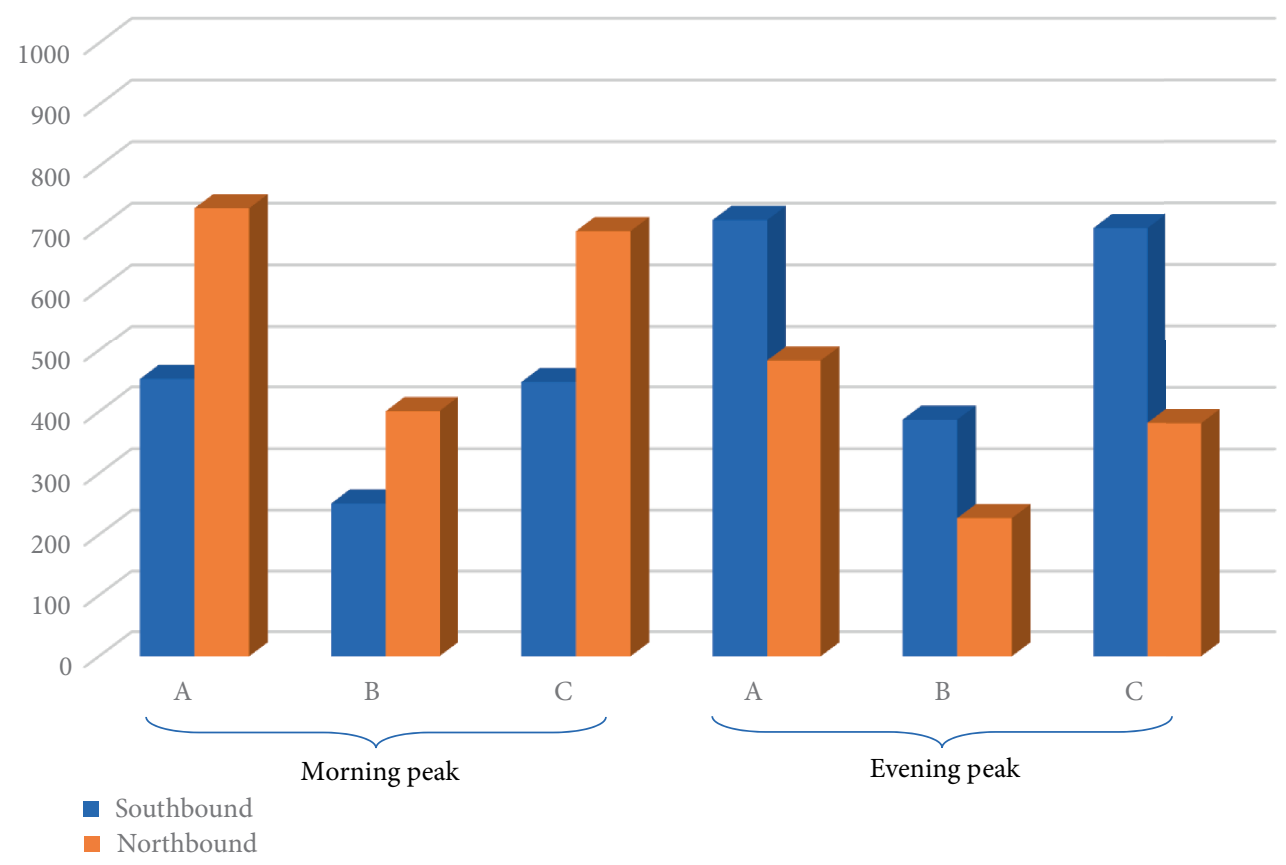

FigURE 3: Hourly traffic volume of South Huaihai road segment in peak time.

TABLE 2: Vehicle speed at North Beijing road segment.

\begin{tabular}{lcccc}
\hline \multicolumn{5}{c}{ Average speed in the morning peak } \\
Average speed & Sedan car & Medium bus & Bus & Truck \\
\hline Northbound & 39.4 & 31.6 & 24.1 & 28.7 \\
Southbound & 35.5 & 28.4 & 16.1 & 26.9 \\
\hline \multicolumn{5}{c}{ Average speed in the evening peak } \\
Average speed & Sedan car & Medium bus & Bus & Truck \\
\hline Northbound & 33.6 & 28.1 & 17.4 & 27.8 \\
Southbound & 38.4 & 32.5 & 23.5 & 29.1 \\
\hline
\end{tabular}

TABLE 3: Vehicle speed at South Huaihai road segment.

\begin{tabular}{lcccc}
\hline \multicolumn{5}{c}{ Average speed in the morning peak } \\
Average speed & Sedan car & Medium bus & Bus & Truck \\
\hline Northbound & 41.1 & 28.5 & 21.3 & 30.1 \\
Southbound & 33.2 & 27.4 & 17.6 & 25.9 \\
\hline \multicolumn{5}{c}{ Average speed in the evening peak } \\
Average speed & Sedan car & Medium bus & Bus & Truck \\
\hline Northbound & 34.1 & 27.9 & 16.5 & 28.1 \\
Southbound & 39.8 & 31.8 & 22.4 & 28.6 \\
\hline
\end{tabular}

4.1.2. Simulation Parameters. Considering the significant traffic tidal phenomenon, we only use VISSIM to simulate the traffic situation in the morning peak. The traffic data needed in the simulation model are required through connected vehicle system and field survey, which are shown in Table 5.

4.2. Simulation Results Analysis. The simulation time is two hours in the morning peak, namely $0-7200 \mathrm{~s}$, the first $90 \mathrm{~s}$ is the warm-up time, and 91-200s is the simulation time where the simulation result is recorded. The simulation results were recorded every $90 \mathrm{~s}$, and we mainly compare the travel time, the number of vehicles passed through the cross section per unit time, and delay before and after the HOV carpooling lane is introduced.

\subsubsection{Simulation Results of North Beijing Road (Bidirectional}

Four-Lane). The simulation results are shown in Figures 9, 10, and Table 6 . Tables 6 and 8 data are derived through VISSIM simulation, where time is the detection time point, No. refers to the detection time interval, Trav is the average travel time, and the other three columns indicate the number of vehicles passed through the road segment in the corresponding time interval under different schemes.

Table 7 shows that the average speed of the regular HOV carpooling scheme has fallen down by $6.5 \%$ compared with the original road, and the traffic volume is decreased by $6.3 \%$. The traffic situation has not been improved. While the average speed of the reversible HOV carpooling scheme has increased by $2.7 \%$ compared to the original road, the traffic volume has increased by $11.1 \%$. The results show that the reversible HOV carpooling scheme has obvious improvement in traffic condition for the four-lane North Beijing road.

\subsubsection{Simulation Results of South Huaihai Road (Bidirectional}

Six-Lane). The simulation results are shown in Figures 11, 12 , and Table 8.

Table 9 shows that for South Huaihai road, the average speed of the regular HOV carpooling scheme has dropped by $2.3 \%$, and the traffic volume decreased by $9.5 \%$ comparing to the original road. The traffic situation has not been improved. However, the reversible HOV carpooling scheme has met an increase of $11.2 \%$ on average speed and $27.8 \%$ on traffic volume compared to the original road. The reversible $\mathrm{HOV}$ 
TABLE 4: Number of different types of vehicles on the selected road segment in peak time.

\begin{tabular}{|c|c|c|c|c|}
\hline \multirow{2}{*}{ Vehicle class } & \multirow{2}{*}{ Number of passengers } & \multicolumn{2}{|c|}{ The ratio of different types of vehicles } & \multirow{2}{*}{$\begin{array}{c}\text { The average ratio of different } \\
\text { types of vehicles }\end{array}$} \\
\hline & & South Huaihai road & North Beijing road & \\
\hline Sedan car & Sedan car with 1 passenger & $64.93 \%$ & $57.74 \%$ & $61.34 \%$ \\
\hline Sedan car & Sedan car with 2 passengers & $23.57 \%$ & $31.55 \%$ & $27.56 \%$ \\
\hline Sedan car & $\begin{array}{c}\text { Sedan car with more than } 3 \\
\text { passengers }\end{array}$ & $4.38 \%$ & $4.53 \%$ & $4.46 \%$ \\
\hline Medium bus & & $1.17 \%$ & $0.41 \%$ & $0.79 \%$ \\
\hline Bus & & $5.07 \%$ & $4.53 \%$ & $4.8 \%$ \\
\hline Truck & & $0.88 \%$ & $1.24 \%$ & $1.06 \%$ \\
\hline
\end{tabular}

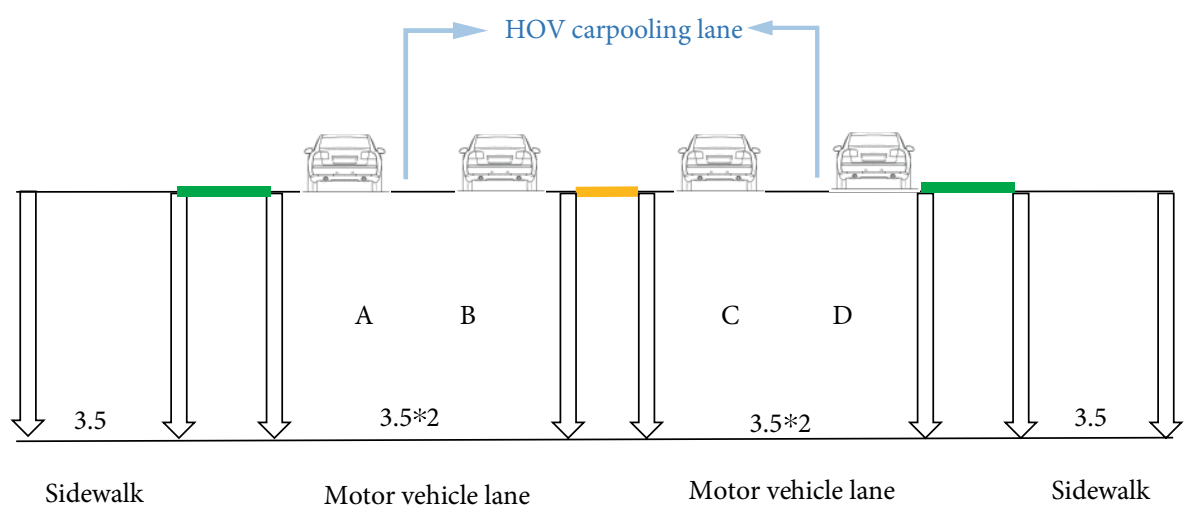

FIGURE 4: Longitudinal configuration of North Beijing road segment.

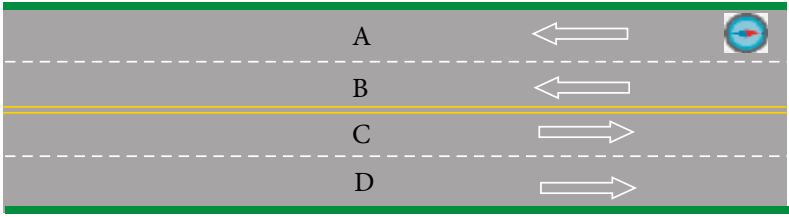

(a)

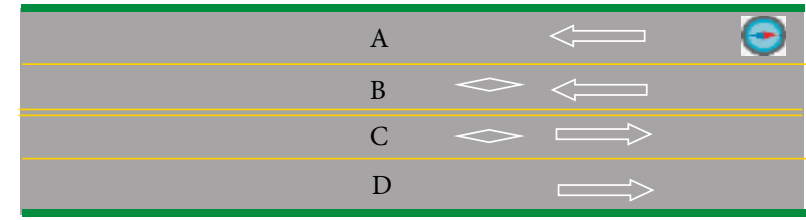

(b)

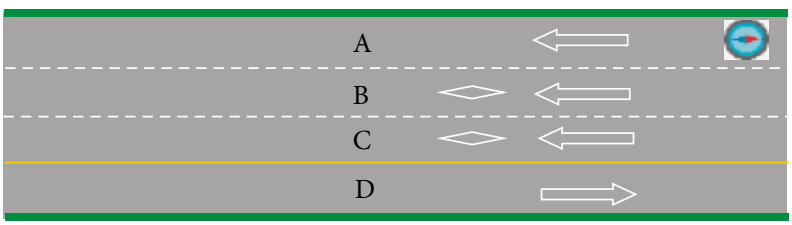

(c)

FIgure 5: Simulation of the North Beijing road before and after the HOV carpooling lane was introduced: (a) The original North Beijing road. (b) Lane B and lane $\mathrm{C}$ were set as the regular HOV lane on North Beijing road. (c) Lane B and lane C were set as the reversible HOV lane on North Beijing road.

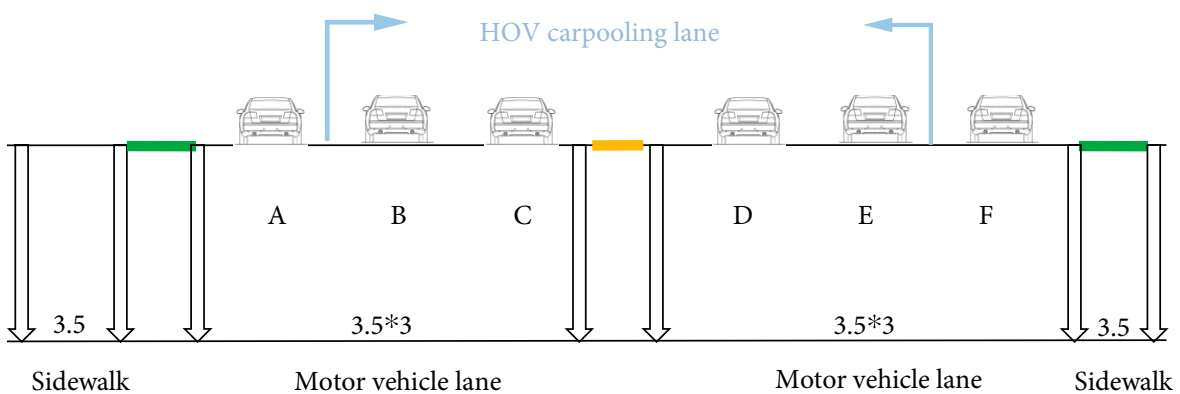

FIGURE 6: Longitudinal configuration of South Huaihai road. 


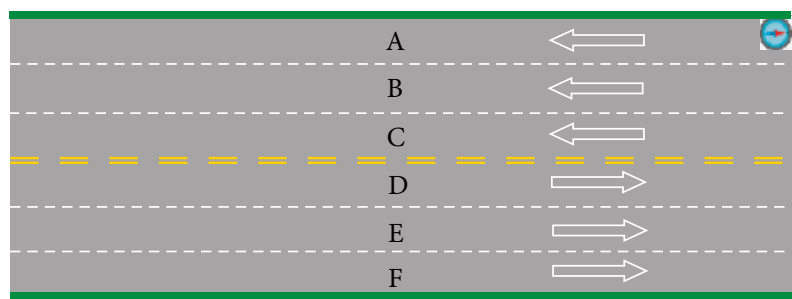

(a)

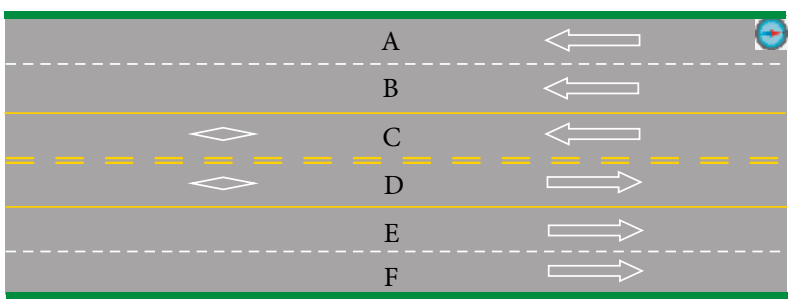

(b)

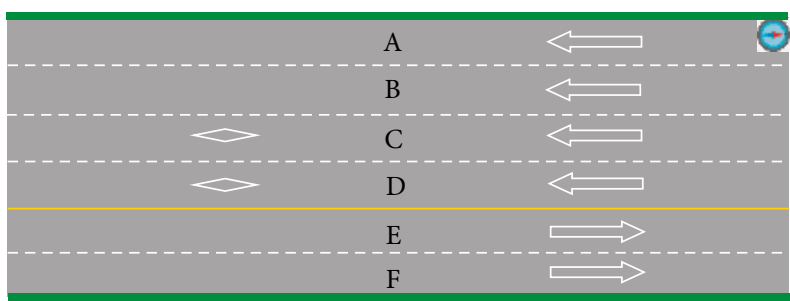

(c)

Figure 7: Simulation of the South Huaihai road before and after the HOV carpooling lane was introduced: (a) The original South Huaihai road. (b) Lane C and lane D were set as the regular HOV lane on South Huaihai road. (c) Lane B and lane C were set as the reversible HOV lane on South Huaihai road.

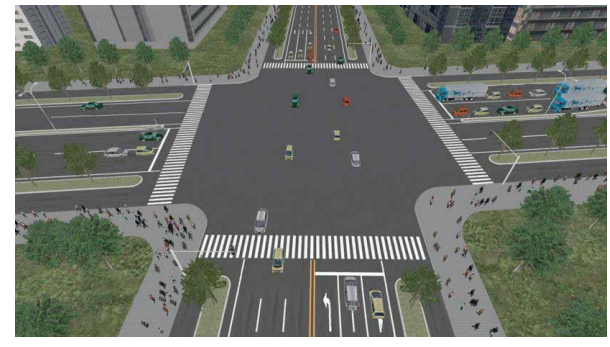

FIGURE 8: Illustration of the selected road segment in VISSIM.
TABLE 5: Parameters in VISSIM simulation.

\begin{tabular}{|c|c|}
\hline Parameters & Setting \\
\hline Segments & North Beijing road, South Huaihai road \\
\hline Traffic facilities & Signal control, traffic signs, etc. \\
\hline Traffic volume & Tables 2 and 3 \\
\hline Speed & Figures 3 and 4 \\
\hline Signal timing & Cycle time $97 \mathrm{~s}$, green time $1-40 \mathrm{~s}$ \\
\hline Simulation time & $\begin{array}{l}0-7200 \mathrm{~s} \text { simulation time, first } 90 \mathrm{~s} \text { is } \\
\text { warm-up time, simulation results are } \\
\text { recorded every } 90 \mathrm{~s}\end{array}$ \\
\hline Simulation content & $\begin{array}{l}\text { Travel time and delay before and after } \\
\text { introducing the HOV carpooling lane }\end{array}$ \\
\hline Evaluation data & $\begin{array}{c}\text { Travel time, number of vehicles passed } \\
\text { through the cross section per unit time, } \\
\text { and delay }\end{array}$ \\
\hline
\end{tabular}

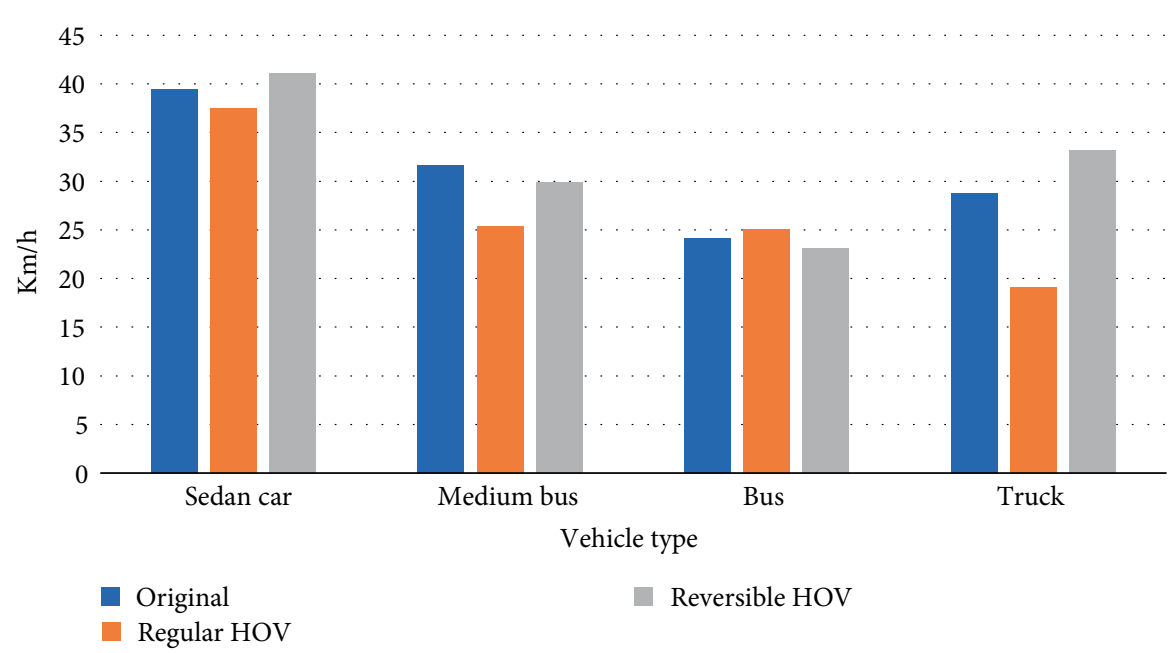

FIGURE 9: Average speed before and after introducing HOV carpooling scheme. 


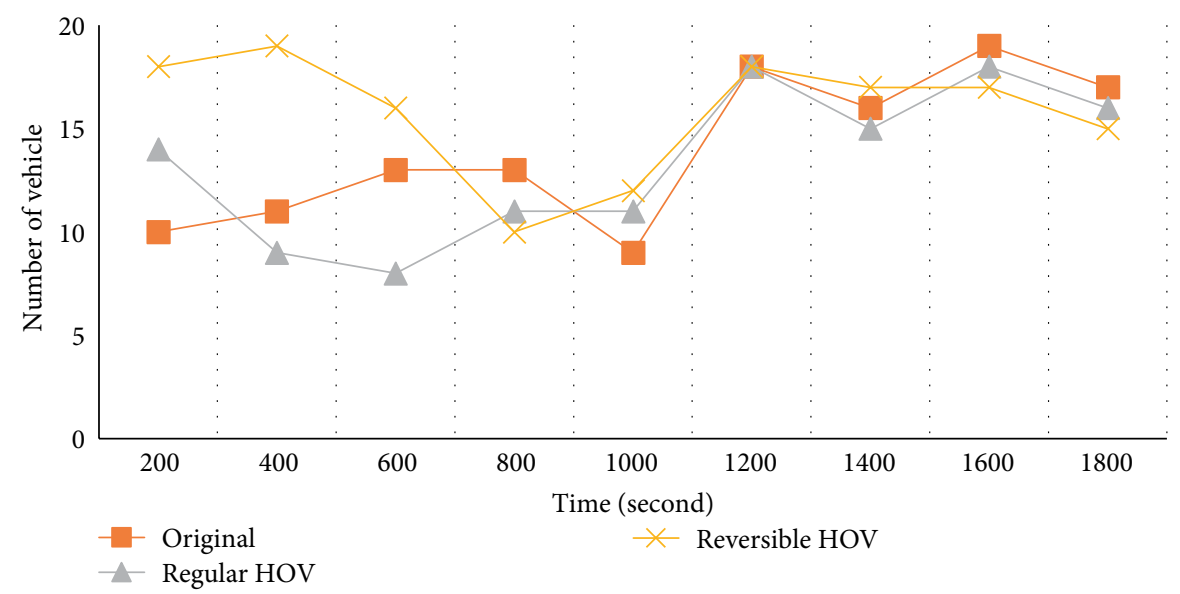

FIGURE 10: Number of vehicles passing through carpooling lanes on North Beijing road.

TABLE 6: Traffic volume before and after optimization of North Beijing road segment.

\begin{tabular}{lccccc}
\hline Time & No & $\begin{array}{c}\text { Original } \\
\text { road }\end{array}$ & $\begin{array}{c}\text { Regular } \\
\text { HOV } \\
\text { scheme }\end{array}$ & $\begin{array}{c}\text { Reversi- } \\
\text { ble HOV } \\
\text { scheme }\end{array}$ & Trav \\
\hline 180 & 24.3 & 10 & 14 & 18 & 23.5 \\
280 & 21.1 & 11 & 9 & 19 & 19.6 \\
370 & 22.5 & 13 & 8 & 15 & 20.3 \\
440 & 22.4 & 13 & 11 & 10 & 22.2 \\
580 & 21.6 & 9 & 11 & 12 & 25.1 \\
610 & 23.1 & 18 & 18 & 15 & 24.4 \\
710 & 25.4 & 16 & 15 & 17 & 24.0 \\
820 & 24.1 & 19 & 17 & 18 & 24.3 \\
880 & 22.7 & 17 & 15 & 16 & 23.7 \\
& & 126 & 118 & 140 & \\
\hline
\end{tabular}

TABLE 7: Comparison of speed and traffic volume of North Beijing road.

\begin{tabular}{lccc}
\hline & Original road & $\begin{array}{c}\text { Regular HOV } \\
\text { scheme }\end{array}$ & $\begin{array}{c}\text { Reversible } \\
\text { HOV scheme }\end{array}$ \\
\hline $\begin{array}{l}\text { Speed }(\mathrm{km} / \mathrm{h}) \\
\text { Traffic volume } \\
\text { (veh/h) }\end{array}$ & 30.95 & 28.93 & 31.8 \\
$\begin{array}{l}\text { Speed change } \\
\text { Traffic volume } \\
\text { change }\end{array}$ & 126 & 118 & 140 \\
\hline
\end{tabular}

carpooling scheme could significantly improve traffic condition of South Huaihai road (bidirectional six-lane) on vehicle speed and traffic volume.
TABLE 8: Traffic flow before and after optimization of South Huaihai road segment.

\begin{tabular}{lccccc}
\hline Time & No & $\begin{array}{c}\text { Original } \\
\text { road }\end{array}$ & $\begin{array}{c}\text { Regular } \\
\text { HOV } \\
\text { scheme }\end{array}$ & $\begin{array}{c}\text { Reversi- } \\
\text { ble HOV } \\
\text { scheme }\end{array}$ & Trav \\
\hline 200 & 24.1 & 12 & 15 & 19 & 23.5 \\
290 & 20.0 & 13 & 12 & 21 & 19.6 \\
380 & 20.8 & 15 & 11 & 18 & 20.3 \\
470 & 22.7 & 17 & 21 & 23 & 22.2 \\
560 & 25.6 & 14 & 11 & 21 & 25.1 \\
650 & 24.9 & 22 & 15 & 19 & 24.4 \\
740 & 24.5 & 19 & 21 & 26 & 24.0 \\
830 & 24.8 & 22 & 18 & 25 & 24.3 \\
920 & 24.2 & 20 & 17 & 21 & 23.7 \\
& & 151 & 141 & 193 & \\
\hline
\end{tabular}

TABLE 9: Vehicle speed and traffic flow comparison on South Huaihai road.

\begin{tabular}{lccc}
\hline & Original road & $\begin{array}{c}\text { Regular HOV } \\
\text { scheme }\end{array}$ & $\begin{array}{c}\text { Reversible } \\
\text { HOV scheme }\end{array}$ \\
\hline $\begin{array}{l}\text { Speed }(\mathrm{km} / \mathrm{h}) \\
\begin{array}{l}\text { Traffic volume } \\
\text { (veh/h) }\end{array}\end{array}$ & 30.25 & 29.55 & 33.63 \\
$\begin{array}{l}\text { Speed compare } \\
\text { Traffic volume } \\
\text { comparison }\end{array}$ & 151 & 141 & 193 \\
\hline
\end{tabular}

4.3. Validity Test. According to the validity test formulas and the actual traffic flow on South Huaihai road, the 


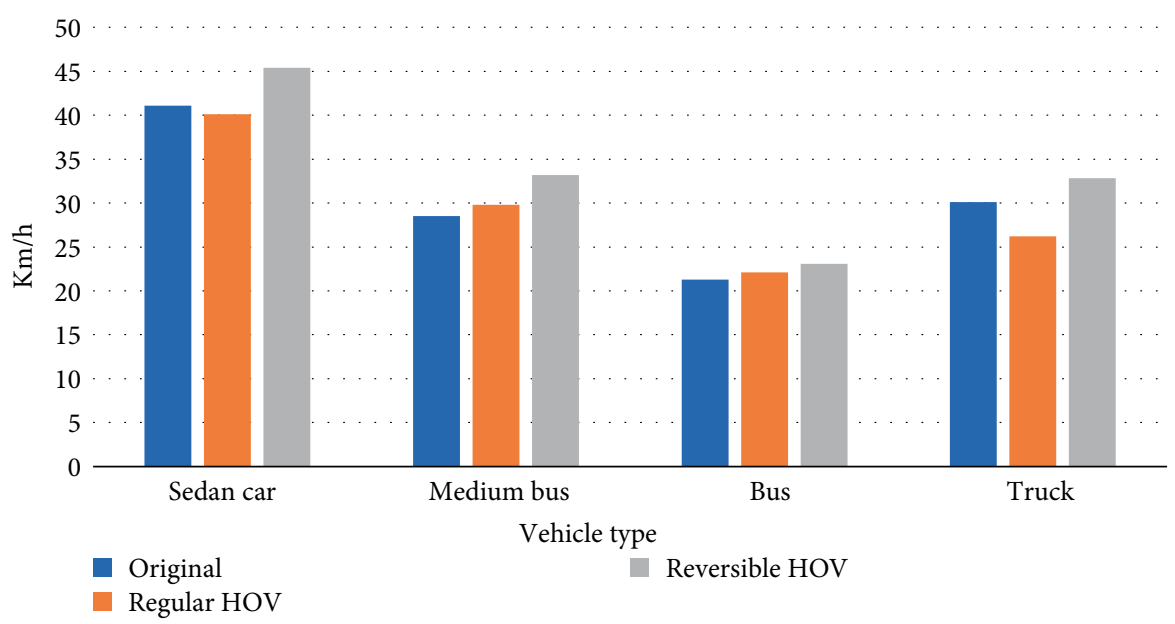

FIGURE 11: Average speed before and after introducing HOV carpooling scheme on South Huaihai road.

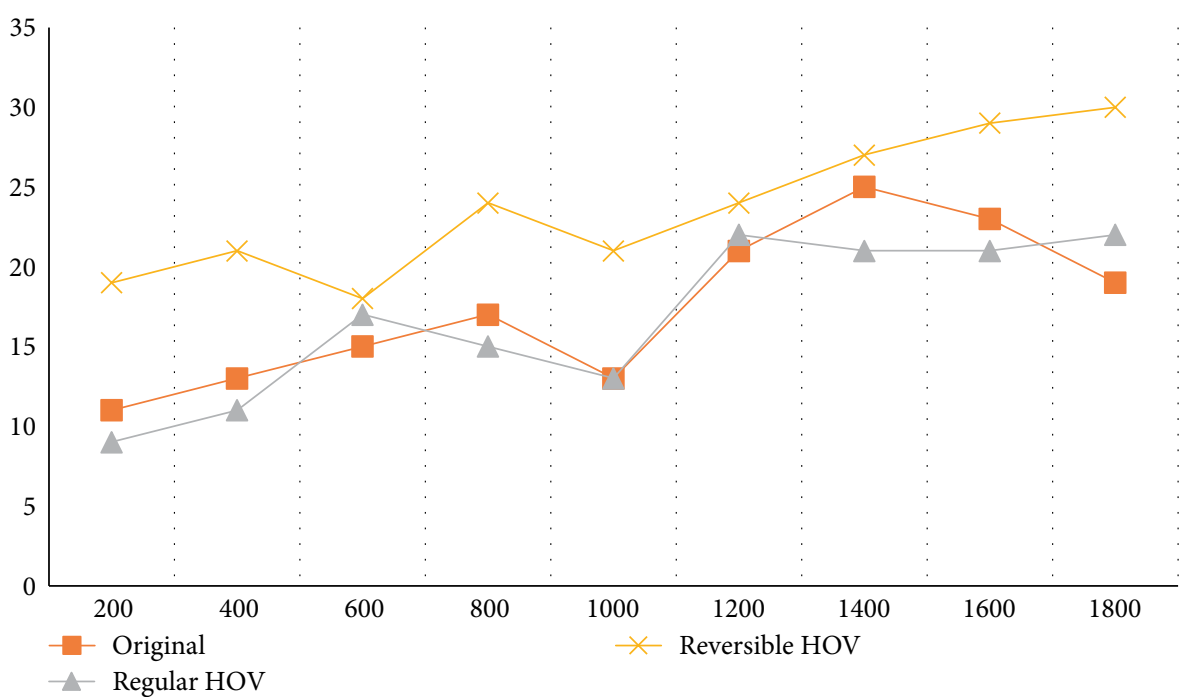

FIGURE 12: Number of vehicles passing through South Huaihai road.

TABLE 10: Average relative error.

\begin{tabular}{lccccc}
\hline Position & $\begin{array}{c}\text { Actual } \\
\text { value }\end{array}$ & $\begin{array}{c}\text { Regular } \\
\text { HOV } \\
\text { scheme }\end{array}$ & $\begin{array}{c}\text { Reversi- } \\
\text { ble HOV } \\
\text { scheme }\end{array}$ & Relative error \\
\hline $\begin{array}{l}\text { South } \\
\text { Huaihai } \\
\text { road }\end{array}$ & 56 & 53 & 62 & $5.6 \%$ & $9.6 \%$ \\
\hline
\end{tabular}

average relative error is calculated based on the traffic flow in 15 minutes.

Table 10 shows that the relative error of the simulation results is less than $10 \%$ compared with the actual situation. The simulation results are effective.

\section{Conclusions}

Under the connected vehicle environment, integrating HOV carpooling lane into the urban arterial roads can make full use of limited traffic facilities and maximize transportation efficiency. The paper proposed two types of HOV carpooling scheme in Huaian (Jiangsu Province): regular HOV carpooling scheme and reversible HOV carpooling scheme. VISSIM was used to test and evaluate the traffic performance (travel time, number of vehicles passed through the cross section per unit time, and delay) on urban arterial roads before and after introducing HOV carpooling schemes. The results showed that the average speed was decreased by $6.5 \%$ and the traffic volume was declined by $6.3 \%$ after introducing regular HOV carpooling scheme on a road with bidirectional four lanes, while the reversible HOV carpooling scheme on the same road resulted in $2.7 \%$ and $11.1 \%$ increment in average speed and traffic volume, respectively. After introducing the regular HOV carpooling scheme on a road with bidirectional six lanes, the average speed was decreased by $2.3 \%$, and the traffic volume fell down by $9.5 \%$. When it comes to the reversible HOV carpooling scheme, the average speed, and the traffic volume were increased by $11.2 \%$ and $27.8 \%$, respectively. Therefore, the reversible HOV carpooling scheme is more suitable for optimizing the urban arterial roads with bidirectional four-lane and six-lane in some old cities. 
Future research should focus on accurate real-time data acquisition from the connected vehicle environment and further supporting the real-time segmentation adjustment of $\mathrm{HOV}$ carpooling lanes. It will help to realize intelligent urban traffic.

\section{Data Availability}

The data used to support the findings of this study are included within the article.

\section{Conflicts of Interest}

The authors declare that they have no conflicts of interest.

\section{Acknowledgments}

Lina Mao conceived and designed the paper; Wenquan Li conducted the model and simulation; Lina Mao and Pengsen $\mathrm{Hu}$ wrote the paper; Guiliang Zhou designed the HOV carpooling schemes; Huiting Zhang analyzed the simulation results; and Xuanyu Zhou collected traffic data. This research was supported by the open fund for Graduate Innovative Projects of Jiangsu Province (KYLX15_0148), the National Natural Science Foundation of China $(61573098,51308246)$, University Natural Science Major Basic Project of Jiangsu Province (15KJA580001), Youth Foundation of Huaiyin Institute of Technology (HGC1408), Jiangsu Government Scholarship for Overseas Studies (JS-2016-K009), Jiangsu Key Laboratory of Traffic and Transportation Security (Huaiyin Institute of Technology) (TTS2016-06). We wish to thank the anonymous reviewers who helped to improve the quality of the paper.

\section{References}

[1] H. Konishi and S.-I. Mun, "Carpooling and congestion pricing: HOV and HOT lanes," Regional Science and Urban Economics, vol. 40, no. 4, pp. 173-186, 2010.

[2] C.-P. Chu, J.-F. Tsai, and S.-R. Hu, "Optimal starting location of an HOV lane for a linear monocentric urban area," Transportation Research Part A: Policy and Practice, vol. 46, no. 3, pp. 457-466, 2012.

[3] S. Shewmake, "Can carpooling clear the road and clean the air? evidence from the literature on the impact of HOV lanes on VMT and air pollution," Social Science Electronic Publishing, vol. 27, no. 4, pp. 363-374, 2012.

[4] I. Stamos, G. Kitis, S. Basbas, and I. Tzevelekis, "Evaluation of a high occupancy vehicle lane in central business district Thessaloniki," Procedia - Social and Behavioral Sciences, vol. 48, pp. 1088-1096, 2012.

[5] J. Zhao, W. Ma, Y. Liu, and X. Yang, "Integrated design and operation of urban arterials with reversible lanes," Transportmetrica B: Transport Dynamics, vol. 2, no. 2, pp. 130$150,2014$.

[6] K. Boriboonsomsin and M. Barth, "Impacts of freeway highoccupancy vehicle lane configuration on vehicle emissions," Transportation Research Part D Transport and Environment, vol. 13, no. 2, pp. 112-125, 2008.
[7] J. Dahlgren, "High occupancy vehicle lanes: not always more effective than general purpose lanes," Transportation Research Part A: Policy and Practice, vol. 32, pp. 99-114, 1998.

[8] J. R. Pooler, W. Robert, and T. Balaker, "Virtual exclusive busways: improving urban transit while relieving congestion," 2005.

[9] C. Wei and B. L. Chen, "HOV will be the priority to introduce China's urban transport planning management," Urban Planning, pp. 93-96, 2003.

[10] J. Zhan, X. D. Pan, and A. Gao, "Design and application of HOV lane," Journal of Transportation and Transportation Technology, pp. 17-20, 2007.

[11] W. L. Wang, Z. D. Yuan, "Study on HOV lane comprehensive evaluation method based on projection pursuit principle," Journal of East China Jiaotong University, pp. 26-32, 2009.

[12] R. Wang, "Shaping carpool policies under rapid motorization: the case of Chinese cities," Transport Policy, vol. 18, no. 4, pp. 631-635, 2011.

[13] R. Avelar, K. Fitzpatrick, K. Dixon, and T. Lindheimer, "The influence of general purpose lane traffic on managed lane speeds: an operational study in Houston Texas," Transportation Research Procedia, vol. 15, pp. 548-560, 2016.

[14] J. Zhao, Y. Liu, and X. Yang, “Operation of signalized diamond interchanges with frontage roads using dynamic reversible lane control," Transportation Research Part C: Emerging Technologies, vol. 51, pp. 196-209, 2015.

[15] M. Zhao, W.-C. Cui, and X. Li, "Multidisciplinary design optimization of a human occupied vehiclse based on Bi-Level integrated system collaborative optimization," China Ocean Engineering, vol. 29, no. 4, pp. 599-610, 2015.

[16] G. Zhang, Y.-J. Wu, X. Liu, and Y. Wang, "Impacts of high occupancy toll lane operations on high occupancy vehicle travelers," in 13th International IEEE Conference on Intelligent Transportation Systems, IEEE, Funchal, Portugal, 2010. 


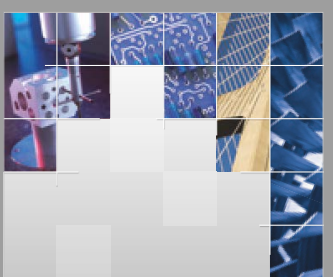

\section{Enfincering}
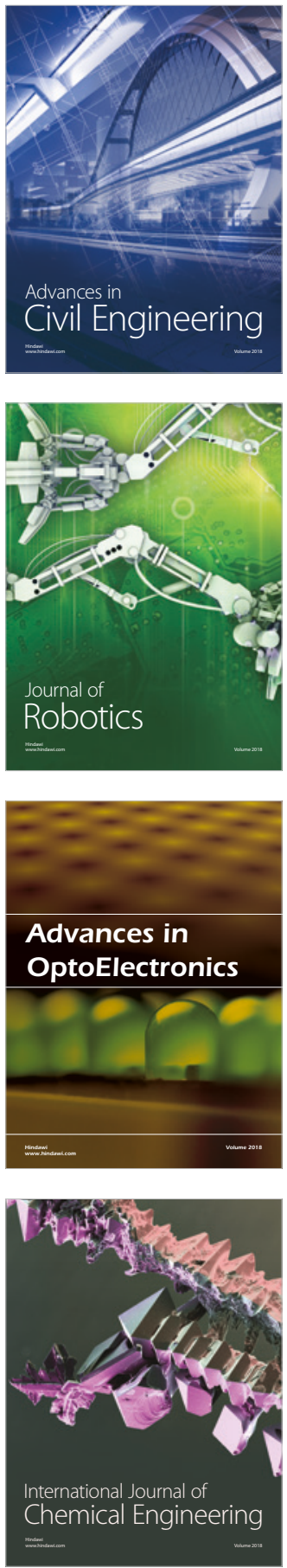

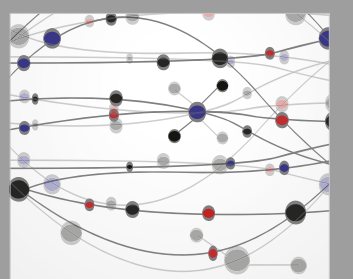

\section{Rotating \\ Machinery}

The Scientific World Journal

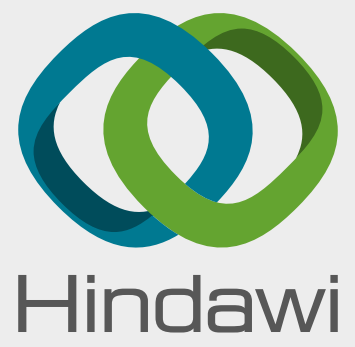

Submit your manuscripts at

www.hindawi.com
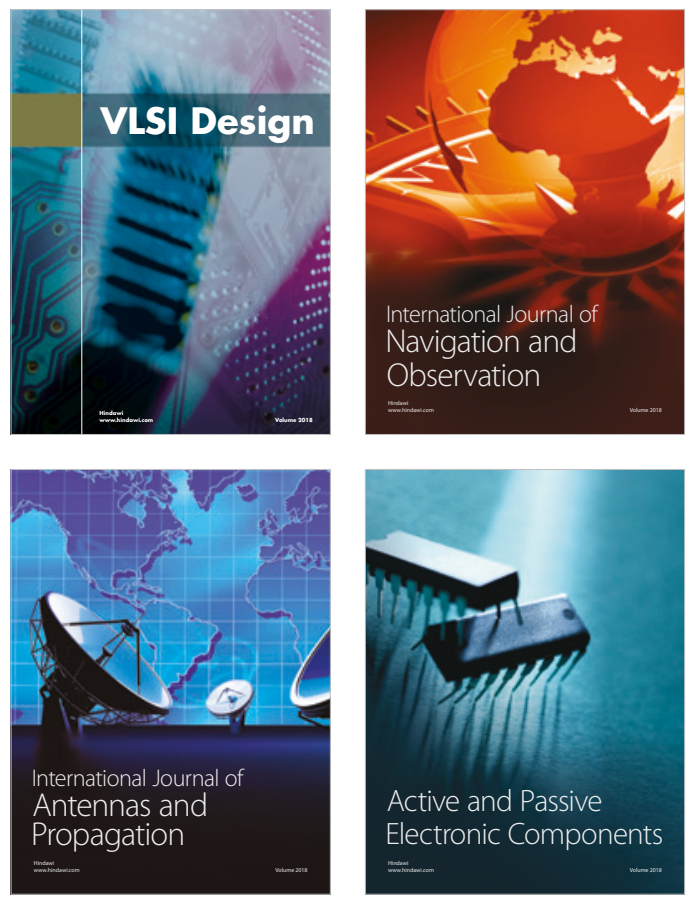
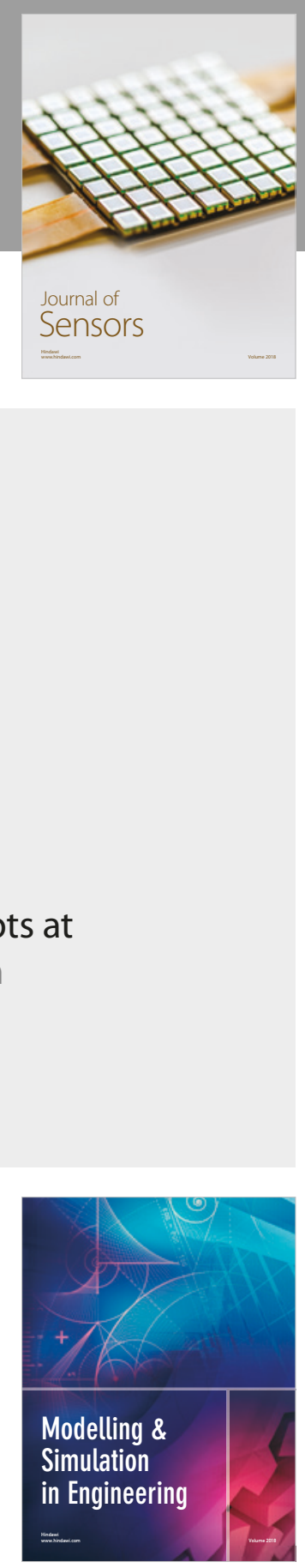

\section{Advances \\ Multimedia}
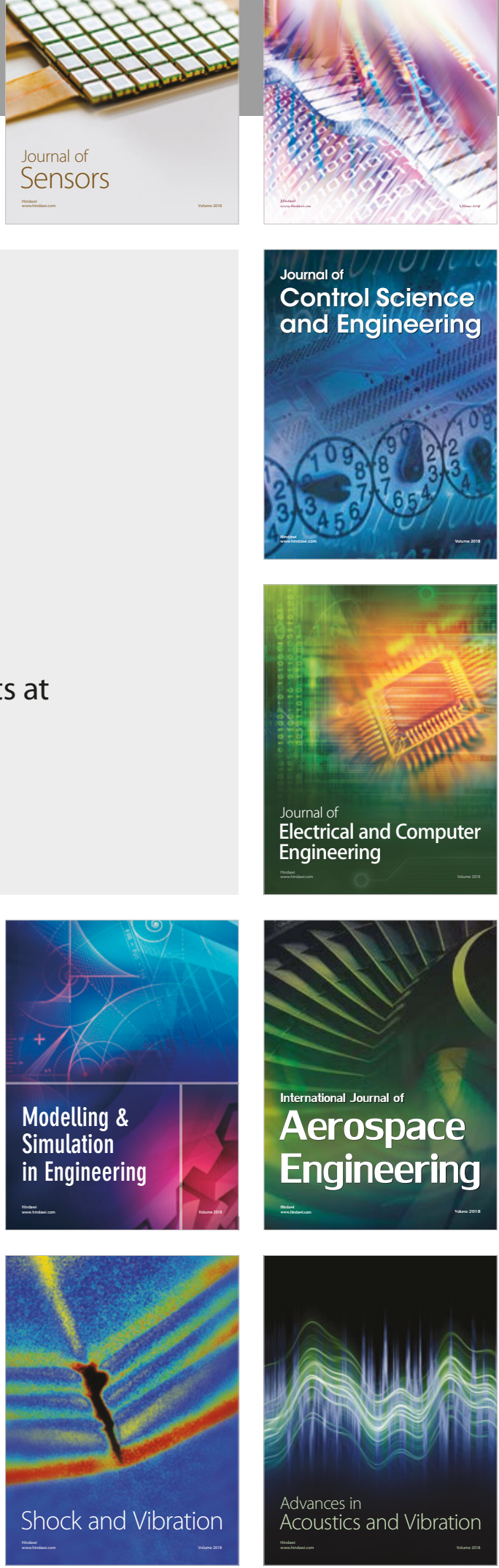